\title{
Thermal Development of Free Volumes in Nafion Membrane
}

\author{
O. Melikhova*, J. ČížEK, L. Košinová And P. HrušKA \\ Faculty of Mathematics and Physics, Charles University, V Holešovičkách 2, 18000, Praha 8, Czech Republic
}

\begin{abstract}
In this work we employed positron lifetime spectroscopy for investigation of the thermal development of free volumes in $\mathrm{H}^{+}$Nafion membrane over a broad range of temperatures from -150 to $150{ }^{\circ} \mathrm{C}$. Positron lifetime studies were combined with differential scanning calorimetry. Size distribution of free volumes was determined from ortho-positronium contribution to positron lifetime spectra. Our investigations revealed that the mean size of free volume holes strongly increases with temperature. On the other hand, the width of the size distribution and the positronium yield decreases with temperature. Transition temperatures corresponding to a change in the slope of the temperature dependence of ortho-positronium lifetime were identified. Results of positron lifetime spectroscopy agree well with the curve obtained by differential scanning calorimetry.
\end{abstract}

DOI: 10.12693/APhysPolA.132.1538

PACS/topics: 78.70.Bj, 61.66.Hq, 82.35.Lr

\section{Introduction}

Nafion is perfluorosulfonated cation exchange membrane developed by du Pont de Nemours \& Co. Inc. Nafion consists of a hydrophobic polytetrafluoroethlylene (PTFE) backbone and hydrophilic perfluoroether side chains terminated by sulfonic acid groups, which readily absorb water and agglomerate into clusters [1]. Nafion exhibits ion conductivity, i.e. proton transport through the membrane is possible, but no electron conductivity. This unique property together with its excellent thermal and chemical stability enables use of Nafion as a proton exchange membrane in electrolyzers and in proton exchange membrane fuel cells (PEMFCs) [2].

Nafion transport properties are closely related to its free volume structure. Positron annihilation spectroscopy (PAS) enables non-destructive determination of size distribution of free volume holes using positronium (Ps) as a probe [3]. Ps formed in polymers is localized in free volumes. There is a correlation between the lifetime of ortho-Ps $(o-\mathrm{Ps})$ and the pore size. This relation is described by well established Tao-Eldrup model $[4,5]$ which has been extended by Ito et al. [6] for large cavities.

Absorption of water in Nafion causes a significant volume expansion (swelling) of the membrane. Previous PAS investigations of free volumes in Nafion [7-10] revealed that water absorbed in Nafion makes size of free volumes smaller and their size distribution narrower. The structure of free volumes changes with temperature. The knowledge of thermal development of free volumes is important since Nafion membrane is subjected to extensive thermal cycling during PEMFC operation. Sodaye et al. [10] studied the effect of temperature on free volumes in Nafion by the Doppler broadening (DB) spectroscopy and found a strong variation of the line-shape $S$ -

\footnotetext{
* corresponding author; e-mail: Oksana.Melikhova@mff.cuni.cz
}

parameter with temperature of the sample. Phase transitions in $\mathrm{H}^{+}$form of Nafion were deduced from changes in the slope of the $S$-parameter temperature dependence, namely a low temperature $\gamma$-transition [11] taking place at $\approx-100^{\circ} \mathrm{C}$ attributed to transition in PTFE backbone of Nafion and a high temperature $\alpha$-transition [11] occurring in the temperature range from 60 to $110^{\circ} \mathrm{C}$ and assigned to the ionic clusters.

Determination of free volume size distribution requires positron lifetime (PALS) spectroscopy. Temperature dependent PALS measurements were performed by Sodaye et al. [10] as well but at a few temperatures only which did not allow determination of phase transition temperatures. Mohamed et al. [12] employed PALS spectroscopy for investigation of thermal development of free volumes in dry Nafion in the temperature range from -60 to $80^{\circ} \mathrm{C}$. A change in slope of the temperature dependence of $o$ Ps lifetime was observed at $\approx 20^{\circ} \mathrm{C}$ and attributed to $\beta$-transition [13] caused by the glass transition in the Nafion backbone. Note that this transition has not been detected by DB spectroscopy performed by Sodaye et al. [10].

Note that only the temperature dependence of the $o$ Ps lifetime has been studied so far. The size distribution of free volume was not considered. In the present work PALS spectroscopy was employed for a detailed investigation of thermal development of free volume size distribution in Nafion in a broad temperature range. The PALS investigations were combined with differential scanning calorimetry (DSC) which provides independent information about phase transitions in Nafion.

\section{Experimental details}

Nafion membrane $\mathrm{N}-1110\left(\mathrm{H}^{+}\right.$form, equivalent weight $1100 \mathrm{~g} / \mathrm{mol}$, thickness $0.254 \mathrm{~mm}$ ) was investigated. As received Nafion membranes were dried at $80^{\circ} \mathrm{C}$ for $1 \mathrm{~h}$ prior measurement to remove absorbed water. Specimens for PALS measurements were multi-stacks consisting of four membranes tightly attached to each other to ensure that all positrons are stopped inside the sample. 
The PALS measurements were performed on a digital spectrometer with time resolution of 145 ps [14]. A ${ }^{22} \mathrm{Na}$ positron source with activity of $1 \mathrm{MBq}$ deposited on $5 \mu \mathrm{m}$ thick Ni foil was sandwiched between two identical specimens. The source contribution with lifetime of 150 ps and intensity of $12 \%$ was always subtracted from PALS spectra. Temperature-dependent PALS measurements were performed in vacuum $\left(10^{-3}\right.$ mbar $)$ in a liquid nitrogen cryostat. Positron source and sample were attached on a $\mathrm{Cu}$ holder plate with a heater and a Pt100 temperature sensor. Temperature of sample during the experiment was controlled with the accuracy of $\pm 0.1^{\circ} \mathrm{C}$. The PALS spectra were decomposed by a least square fitting program PALS (version 9) [15].

The DSC and thermogravimetry (TG) studies were performed on a simultaneous DSC/TG apparatus Setaram Labsys Evo. The DSC measurements were performed in an $\mathrm{Al}$ crucible with the heating rate of $20 \mathrm{~K} / \mathrm{min}$ in dynamic $\mathrm{N}_{2}$ flow (flowing rate $20 \mathrm{ml} / \mathrm{min}$ ).

\section{Results and discussion}

As received Nafion membrane contains water absorbed from air. The concentration of absorbed water depends on the relative humidity in the environment [2]. To study a well defined state of Nafion the as received membrane was firstly dried at $80^{\circ} \mathrm{C}$ for $1 \mathrm{~h}$ to remove absorbed water. Figure 1 shows the relative weight loss of Nafion during drying at $80^{\circ} \mathrm{C}$ determined by weighting of the membrane dried for various time periods (points) and by TG (solid line). Both techniques yielded similar results. The water content in the as-received Nafion was $\approx 6 \mathrm{wt} \%$ and the absorbed water was completely released from the membrane by drying at $80^{\circ} \mathrm{C}$ for approximately $6 \mathrm{~min}$.

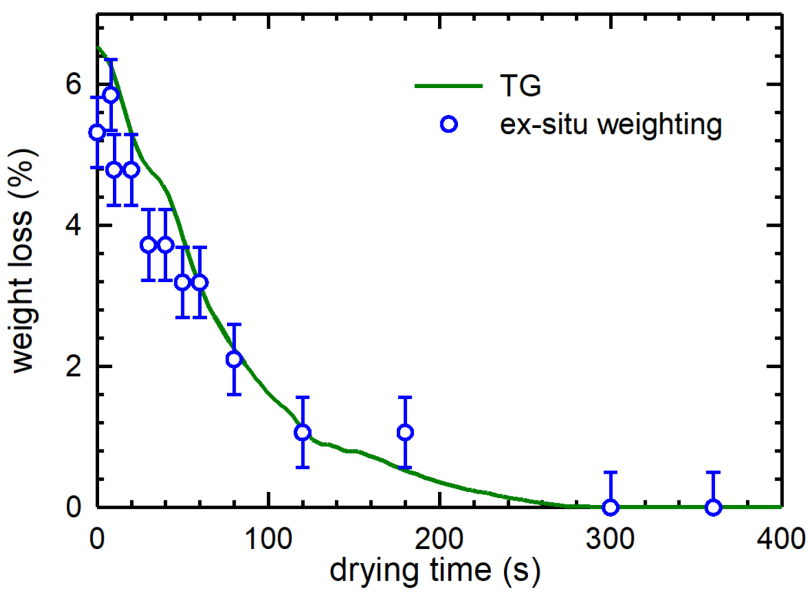

Fig. 1. The relative weight loss of as-received Nafion membrane during drying at $80^{\circ} \mathrm{C}$. The weight loss was determined by exsitu weighting (blue circles) and on other sample by TG.

The PALS spectra of Nafion membrane consisted of two exponential components representing contributions of positrons annihilated as particles and a Ps contribution. The temperature dependence of the positron lifetimes is plotted in Fig. 2a. The shorter and weak

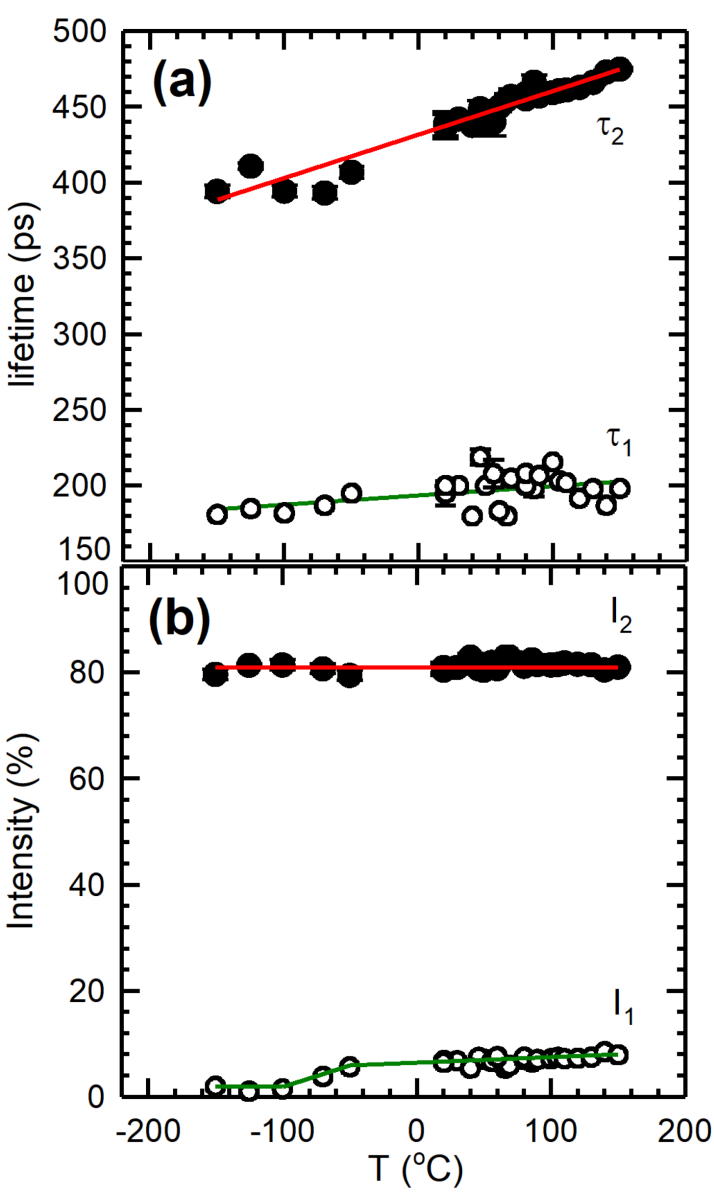

Fig. 2. Temperature dependence of (a) positron lifetimes $\tau_{1}, \tau_{2}$ and (b) corresponding relative intensities for Nafion.

component with lifetime $\tau_{1}$ represents a contribution of delocalized positrons and/or positrons trapped at small open volumes. The longer component with lifetime $\tau_{2}$ and dominating intensity comes from trapped positrons. Both lifetimes $\tau_{1}$ and $\tau_{2}$ obviously increase with temperature reflecting expansion of Nafion structure. Figure $2 \mathrm{~b}$ shows the development of relative intensities $I_{1}$ and $I_{2}$ of the positron components. The contribution of trapped positrons with intensity $I_{2} \approx 80 \%$ is the dominating component in the PALS spectra. The intensity $I_{2}$ does not change with temperature while $I_{1}$ slightly increases in the range from -150 to $0{ }^{\circ} \mathrm{C}$. This is caused by decrease of intensity of the Ps contribution.

The Ps contribution was considered as a complex component consisting of (i) a discrete exponential component with lifetime of $\tau_{p-P s}=125$ ps representing parapositronium ( $p$-Ps) contribution and (ii) a continuous lognormal distribution of annihilation rates arising from $o-$ Ps annihilation in nanoscopic holes of various sizes. In fitting of PALS spectra the intensities of the components (i) and (ii) were kept in the ratio 1:3 corresponding to the branching ratio of $p$-Ps and $o$-Ps. Results of fitting are presented in Fig. 3.

The temperature dependence of the mean $o$-Ps life- 


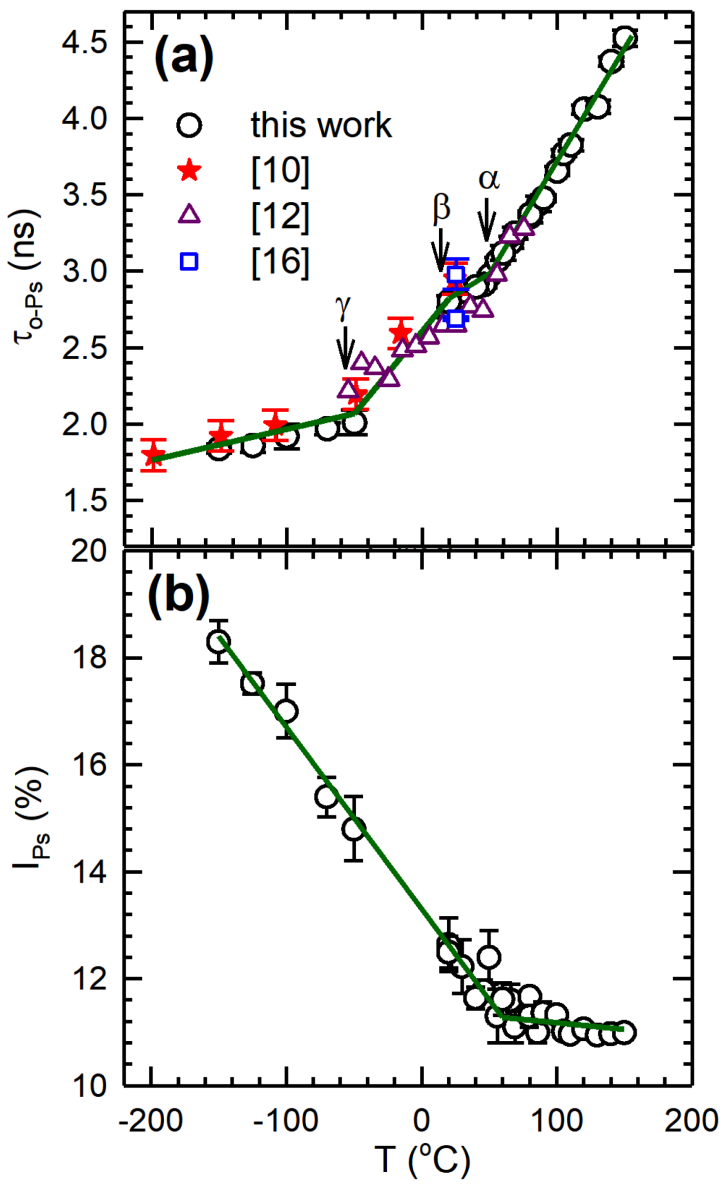

Fig. 3. Temperature dependence of the parameters of $o$-Ps contribution: (a) o-Ps lifetime, (b) intensity of Ps contribution.

time $\tau_{o-P s}$ is plotted in Fig. 3a. The $o$-Ps lifetimes for Nafion reported in literature $[10,12,16]$ are plotted in the figure as well. One can conclude that agreement among results obtained by various authors is satisfactory. The lifetime $\tau_{o-P s}$ increases with temperature reflecting increasing size of free volumes due to increasing magnitude of vibration motion of polymer chains. The increase with temperature is linear with several changes in slope occurring at temperatures where phase transitions in Nafion take place. The phase transition temperatures determined from the development of the $o$-Ps lifetime are summarized in Table I. The $\alpha$-transition [11] observed at $\approx 60^{\circ} \mathrm{C}$ takes place in the ionic cluster region of the polymer [10]. The $\beta$-transition [13] was found at $\approx 20^{\circ} \mathrm{C}$. It has been reported in literature that the $\beta$-transition in Nafion occurs the temperature range from -20 to $20^{\circ} \mathrm{C}$ depending on the water content $[1,13]$ and was attributed to PTFE backbone chain motions within the framework of a static physically crosslinked network [13]. The $\gamma$-transition was determined at $\approx-50{ }^{\circ} \mathrm{C}$. Mechanical relaxation studies performed by Eisenberg and Yeager [11] revealed that the $\gamma$-transition is independent of the cationic form of Nafion and occurs, thereby, inside the PTFE backbone. This interpre- tation is in agreement with DB measurements by Soday et al. [10] which confirmed that the $\gamma$-transition is associated with re-arrangement of free volume holes in the Nafion skeleton. Note that Sodaye et al. [10] observed the $\gamma$-transition at $-100^{\circ} \mathrm{C}$, i.e. at lower temperature than in the present study. The origin of this discrepancy is not clear yet but it could be caused by the fact low temperature DB studies by Sodaye et al. [10] were performed on $\mathrm{H}^{+}$form of Nafion containing water. It has to be mentioned that Boyle et al. [17] performed NMR study of hydrated Nafion and found that absorbed water vitrifies rather than freezes below $0{ }^{\circ} \mathrm{C}$. Subsequently a low temperature phase transition appeared at $-100^{\circ} \mathrm{C}$ which can be attributed to glass transition of water molecules in ionic clusters [17]. This phase transition is obviously absent in our samples since we measured dry Nafion.

TABLE I

The phase transition temperatures determined in $\mathrm{H}^{+}$ form of Nafion by PALS spectroscopy. Results obtained in this work are compared with data available in literature.

\begin{tabular}{c|c|c}
\hline \hline Transition & $T\left[{ }^{\circ} \mathrm{C}\right]$, this work & $T\left[{ }^{\circ} \mathrm{C}\right]$, literature \\
\hline$\alpha$ & $60 \pm 5$ & $60-110[10]$ \\
$\beta$ & $20 \pm 5$ & $20[12]$ \\
$\gamma$ & $-50 \pm 10$ & $-98[10]$
\end{tabular}

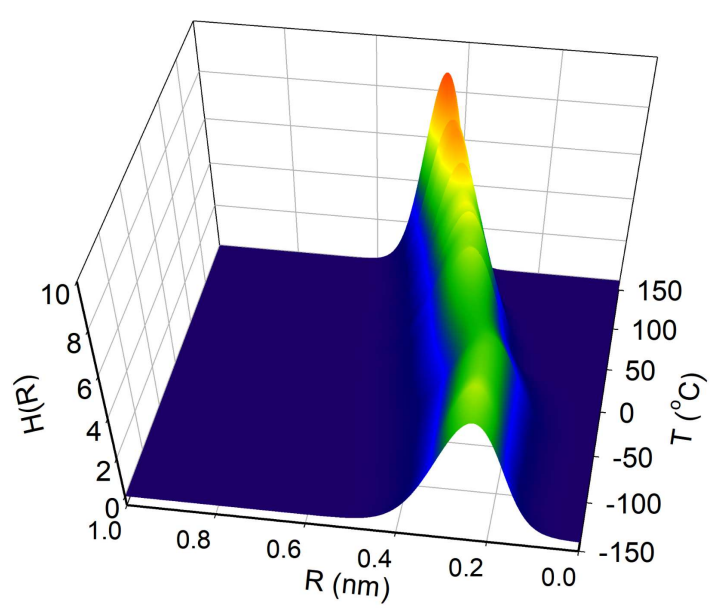

Fig. 4. Thermal development of the free volume size distribution in Nafion.

The development of the intensity of the Ps contribution, $I_{P s}$, is shown in Fig. 3b. One can see in the figure that Ps yield decreases with temperature and saturates at the temperature corresponding approximately to the $\alpha$-transition temperature. The intensity of Ps contribution is influenced not only by the concentration of free volume holes but also by Ps yield which depends on temperature and on variations in chemical environment [18]. An increase of Ps yield is commonly observed at low temperatures and is attributed to Ps formation from shallow trapped electrons [19-21]. 


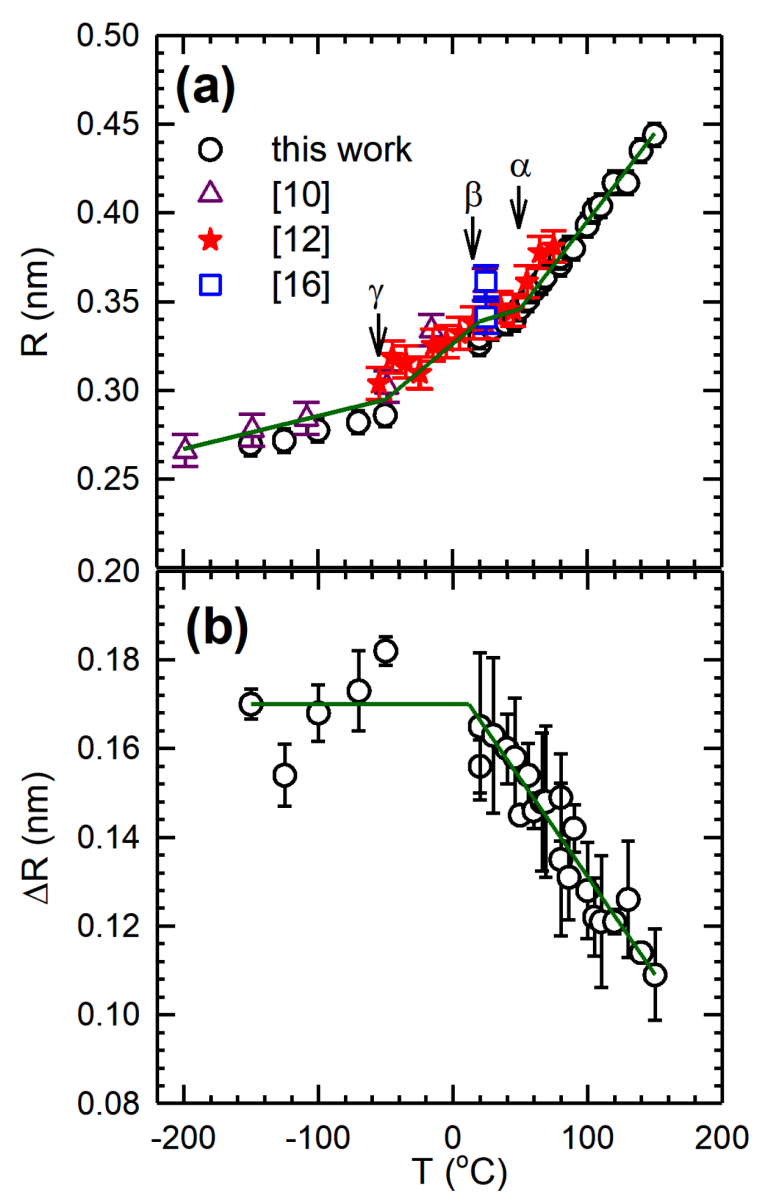

Fig. 5. (a) The mean radius and (b) width (FWHM) of the size distribution of free volumes plotted as a function of temperature.

Figure 4 shows the thermal development of free volume size distribution $H(R)$, where $R$ is the free volume hole radius, calculated from PALS results using the Tao-Eldrup model $[4,5]$. Figure 5a shows the mean radius of free volume holes as a function of temperature. The thermal development of the width (FWHM) of the free volume size distribution is plotted in Fig. 5b. The mean radius of free volume holes obviously increases with temperature reflecting increasing flexibility of polymer chains. The width of the size distribution remains approximately constant up to the $\beta$-transition, at higher temperatures the width of the size distribution decreases. Ps is formed predominantly in the PTFE backbone. The formation of Ps in ionic clusters is suppressed since free electrons in spur created during positron thermalization are caught by sulfonic radicals with uncompensated negative charge. The determined free volume size distribution corresponds, therefore, mainly to the PTFE backbone.

The DSC curve for dried Nafion membrane is shown in Fig. 6. It exhibits an endothermic peak with maximum at $68^{\circ} \mathrm{C}$ which agrees well with the $\alpha$-transition temperature determined by PALS spectroscopy. Heating to higher temperatures leads to two additional endother-

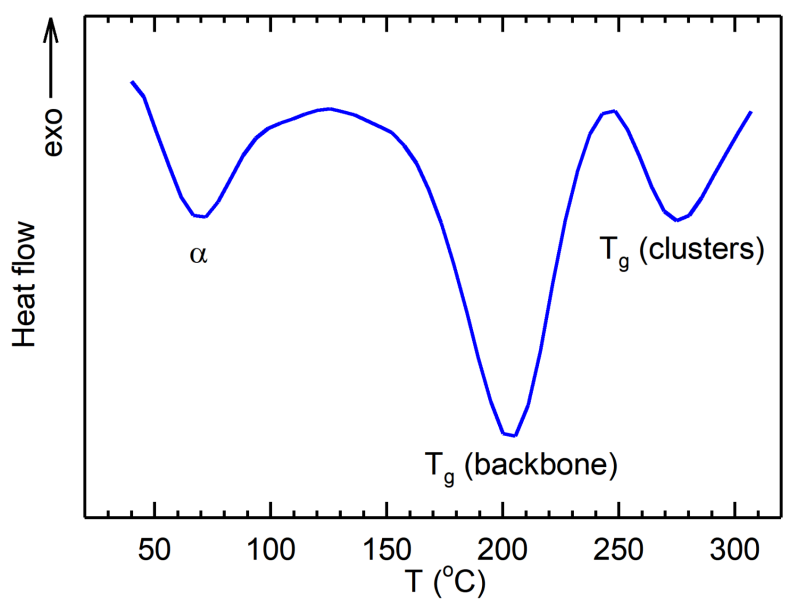

Fig. 6. DSC curve for dried Nafion.

mic processes occurring in the temperature range 150 $235^{\circ} \mathrm{C}$ and $250-300{ }^{\circ} \mathrm{C}$. These endothermic peaks can be attributed to glass transitions occurring in the polymeric PTFE backbone and inside the ionic clusters, respectively $[22,23]$. Heating to temperatures above $300^{\circ} \mathrm{C}$ leads to thermal decomposition of Nafion [24].

\section{Conclusions}

The thermal development of the size distribution of free volumes in dried $\mathrm{H}^{+}$form of Nafion was determined by positron lifetime spectroscopy in a broad temperature range from -150 to $150^{\circ} \mathrm{C}$. Positronium is formed mainly in the hydrophobic Nafion backbone. The mean size of free volume holes increases with temperature due to increasing motion of polymer chains. Three phase transitions were determined as changes in the slope of temperature dependence of the $o$-Ps lifetime: (i) low temperature $\gamma$-transition at $-50^{\circ} \mathrm{C}$ occurring in the Nafion backbone; (ii) the $\beta$-transition at $20^{\circ} \mathrm{C}$ taking place in the Nafion backbone as well, and (iii) the $\alpha$-transition at $60^{\circ} \mathrm{C}$ which occurs inside the ionic clusters. The $\alpha$-transition was observed also by DSC. Two additional endothermic processes taking place in the temperature range $150-235^{\circ} \mathrm{C}$ and $250-300^{\circ} \mathrm{C}$ were determined by differential scanning calorimetry. These processes can be attributed to the glass transition in the polymeric PTFE backbone and inside the ionic clusters, respectively.

\section{Acknowledgments}

This work was supported by the Czech Science Agency (project P108/12/G043).

\section{References}

[1] K.A. Mauritz, R.B. Moore, Chem. Rev. 104, 4535 (2004).

[2] H. Ito, T. Maeda, A. Nakano, H. Takenaka, Int. J. Hydrogen Energy 36, 10527 (2011).

[3] Y.C. Jean, Microchem. J. 42, 72 (1990).

[4] S.J. Tao, J. Chem. Phys. 56, 5499 (1972).

[5] M. Eldrup, D. Lightbody, J.N. Sherwood, Chem. Phys. 63, 51 (1981). 
[6] K. Ito, H. Nakanishi, Y. Ujihira, J. Phys. Chem. B 103, 4555 (1999).

[7] H.S. Sodaye, P.K. Pujari, A. Goswami, S.B. Manohar, J. Polym. Sci. B Polym. Phys. 35, 771 (1997).

[8] H.S. Sodaye, P.K. Pujari, A. Goswami, S.B. Manohar, J. Polym. Sci. B Polym. Phys. 36, 983 (1998).

[9] J. Čížek, Z. Barnovská, M. Šourek, I. Procházka, Mater. Sci. Forum 733, 49 (2013).

[10] H.S. Sodaye, P.K. Pujari, A. Goswami, S.B. Manohar, Radiat. Phys. Chem. 58, 567 (2000).

[11] A. Eisenberg, H.L. Yeager, ACS Symposium Series 180, American Chemical Society, Washington 1982.

[12] H.F.M. Mohamed, K. Ito, Y. Kobayashi, N. Takimoto, Y. Takeoka, A. Ohira, Polymer 49, 3091 (2008).

[13] S.J. Osborn, M.K. Hassan, G.M. Divoux, D.W. Rhoades, K.A. Mauritz, R.B. Moore, Macromolecules 40, 3886 (2007).

[14] F. Bečvář, J. Čížek, I. Procházka, J. Janotová, Nucl. Instrum. Methods Phys. Res. A 539, 372 (2005).

[15] J. Kansy, Nucl. Instrum. Methods Phys. Res. A 374 235 (1996).
[16] R.C. McDonald, D.W. Gidley, T. Sanderson, R.S. Vallery, J. Membrane Sci. 332, 89 (2009).

[17] G. Boyle, J.M.D. Coey, V.J. McBrierty, Chem. Phys. Lett. 86, 16 (1982).

[18] F.H.J. Maurer, M. Schmidt, Radiat. Phys. Chem. 58, 509 (2000).

[19] Y. Ito, T. Hirade, E. Hamada, T. Suzuki, Y. Ito, Acta Phys. Pol. A 95, 533 (1999).

[20] A. Uedono, T. Kawano, S. Tanigawa, M. Ban, M. Kyoto, T. Uozumi, J. Polym. Sci. B 35, 1601 (1997).

[21] C.L. Wang, T. Hirade, F.H.J. Maurer, M. Eldrup, J. Chem. Phys. 108, 4654 (1998).

[22] S. Su, K.A. Mauritz, Macromolecules 27, 2079 (1994).

[23] R.B. Moore, C.R. Martin, Macromolecules 22, 3594 (1989).

[24] S.H. de Almeida, Y. Kawano, J. Therm. Anal. Calorim. 58, 569 (1999). 